\title{
Development of A Road Surface Discrimination Device for Preventing Falls during Winter Walking - Built-In to Shoes and Verification on Frozen Surfaces -
}

\author{
Satoshi Izumiya, Keisuke Kon, Hiroki Matsuzaki, Tamotsu Mitamura
}

\begin{abstract}
In areas with cold winters, snow worsens road surface conditions and causes icy roads that are likely to induce falls. Pedestrians need to use anti-slip products and observe the road surface to avoid falling. However, because it is unrealistic to constantly walk attentively, people exhibit only temporary alertness and cautiousness. Therefore, we developed smart shoes that aid in road surface perception. In this study, we manufactured a device to obtain necessary road surface information, in an effort to clarify whether the surface characteristics of a frozen road surface can be obtained when the device is incorporated into the shoes. In addition, we examined whether useful indicators had been obtained to determine the road surface.

The participants were three healthy adult men without any difficulties in walking. The measurement was performed in the winter as the participants walked on an outdoor frozen road.

The differences between the dry and frozen road surfaces tended to appear at the end of the swing phase, as seen in the road surface information results (reflected light and road surface temperature).

After fabricating a device capable of obtaining road surface information and performing walking experiments on actual frozen surfaces, the results suggest that road surface information can be obtained, even during wintertime walking. This device also enables the acquisition of useful indicators for road surface determination.
\end{abstract}

Index Terms-Frozen road surface, Prevention of falls, Infrared measurement.

\section{INTRODUCTION}

During winter, the repeated melting and freezing of snow in areas with colder climates cause the road conditions to deteriorate, often forming a slippery frozen surface [1]. During this period, the frozen road surface exposes pedestrians to a higher risk of accidental falls [2]. Fall prevention is crucial, as even a single fall can lead to unexpected severe injuries. Particularly, at present, there has been an increase in reports of decreased movement function and balance ability experienced by the elderly population; thus, this can be considered an urgent problem [3-6].

Efforts have been made to devise shoes, canes, and walking methods that make slipping more difficult to prevent such a

Satoshi Izumiya, Division of Engineering, Graduate School of Engineering, Hokkaido University of Science, Sapporo, Japan.

Keisuke Kon, Department of Prosthetics and Orthotics, Faculty of Health Sciences, Hokkaido University of Science, Sapporo, Japan.

Hiroki Matsuzaki, Department of Information and Computer Science, Faculty of Engineering, Hokkaido University of Science, Sapporo, Japan.

Tamotsu Mitamura, Department of Media Arts and Design, Faculty of Future Design, Hokkaido University of Science, Sapporo, Japan. problem [7]. However, as mentioned earlier, the number of falling accidents has not decreased. Therefore, this study focuses on the causes of falls during the winter period. According to previous studies, one of the leading causes of falls is the inability of pedestrians to evaluate the road surface properly [8]. Therefore, the expectation exists that the statistics would improve if the road conditions are identifiable while walking.

Nevertheless, an effective method to perform this evaluation has not yet been established. To detect freezing conditions, road surface measuring equipment, which emits infrared rays and reports temperature changes, mounted on a vehicle or at a fixed point has been previously employed [9]. However, devices that focus on the road used by cars are not optimized for measurement of the sidewalk where pedestrians mainly walk. Moreover, even if the application of existing technology to the sidewalk is considered, the environment, including the power source, space, and sustainability, presents a problem due to its differences from the path of the car.

Therefore, we devised a road surface assessment system that assumes pedestrian use. This system aims to solve the problem mentioned above by measuring road surface characteristics during walking, evaluating the surface using the acquired data, and communicating the determined result to the pedestrian. We previously manufactured a device that enables road surface measurement while walking and evaluated it on an indoor reproduction of a winter road surface [10]. However, the efficiency of the device in a real environment is unknown, as the device was not evaluated on an actual frozen road surface. Therefore, we conducted measurements on a newly frozen outdoor road surface and subsequently reported the obtained results and necessary considerations.

\section{MethoD}

\section{A. Participant Information \& Informed Consent}

The participants were three healthy men in their 20s with no history of orthopedic disease. Participant information is provided in Table 1.

The experiments conducted in this study were approved by the Ethics Committee of the Hokkaido University of Science (approval number 186). In this report, we are getting informed consent from them. 
Table 1 Participant information

\begin{tabular}{lrr}
\hline & \multicolumn{1}{c}{ Avg. } & \multicolumn{1}{c}{ SD. } \\
\hline Age $(\mathrm{y})$. & $23.0 \pm$ & 1.0 \\
Height $(\mathrm{cm})$ & $171.3 \pm$ & 3.5 \\
Weight $(\mathrm{kg})$ & $73.3 \pm$ & 12.7 \\
Foot Length $(\mathrm{cm})$ & $25.3 \pm$ & 1.2 \\
Foot Width $(\mathrm{cm})$ & $9.9 \pm$ & 0.6 \\
Heel Width $(\mathrm{cm})$ & $6.2 \pm$ & 0.6 \\
\hline
\end{tabular}

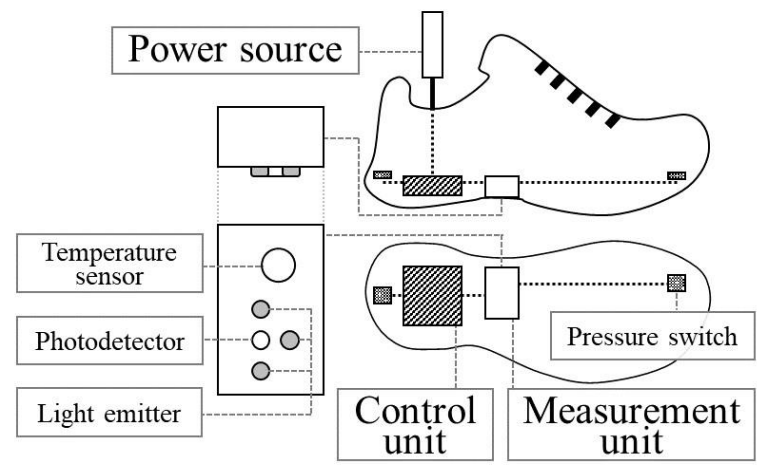

Fig. 1 Device configuration

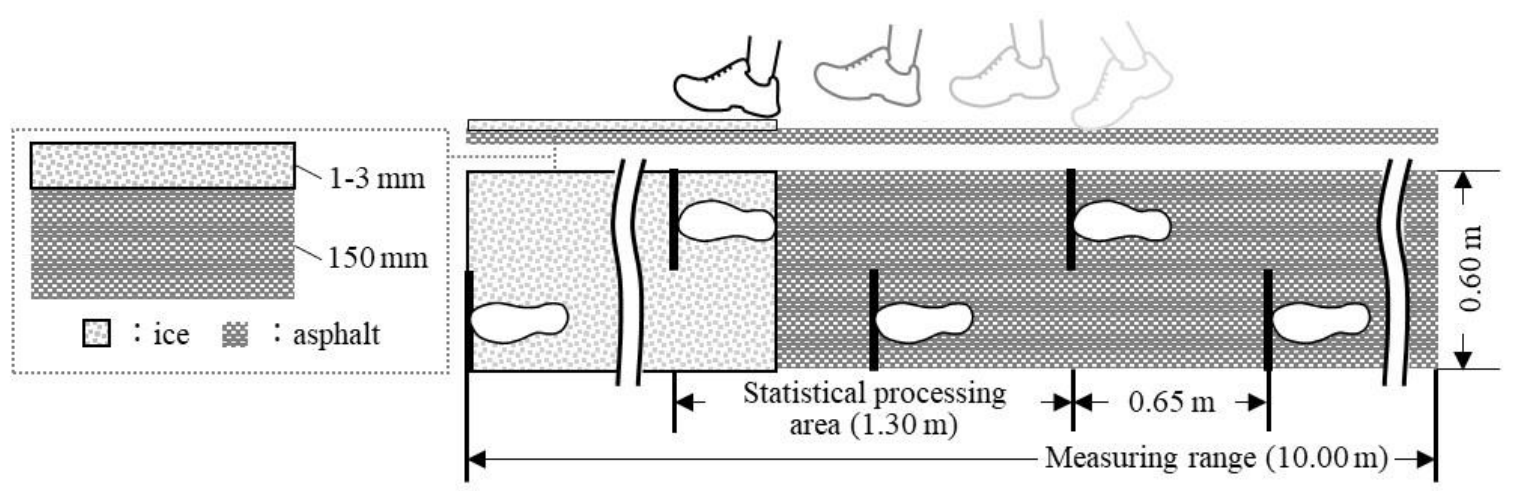

Fig. 2 Configuration of walking path (road condition)

\section{B. Measurement Parameters}

This experiment addresses the question of whether measuring road characteristics while walking outdoors in the winter is possible and whether the measurements obtained are useful in distinguishing between dry and frozen road surfaces. The measurement method used near-infrared light (wavelength $1450 \mathrm{~nm}$ ) to measure the road surface temperature, as roads exhibit high absorption characteristics when covered with the ice layer and water film of the frozen surface [11-12]. The pressures at the toe and heel were measured simultaneously to identify the walking cycle.

\section{Measuring Device}

This study used a shoe-embedded measuring device (after this referred to as "the unit," shown in Fig. 1) to obtain the measurements discussed above [10]. The sampling frequency was $100 \mathrm{~Hz}$. The measurement unit included a light-emitting element (L10660 - 01, HAMAMATSU) that irradiated the road surface with near-infrared light, as well as an element (G12180-010A, HAMAMATSU) that received the reflected light from the road surface. In addition, a temperature sensor (D6T-1A-02, OMRON) was incorporated into the device to measure the surface temperature of the road. The control unit contained a small microcomputer board (Arduino Micro) that processed information from the pressure switches (TSHA_T_2.5B2_19E, Top_Up Industry) installed at the toe and heel, in addition to the amount of light and temperature data obtained by the measuring unit. The data were stored using an SD module (MM-TXS02, Sunhayato). Power was supplied to each component by a mobile battery (A1104011, Anker) attached to the side of the lower leg by a band. The unit was placed on only one side (right foot).

\section{Measuring Method}

The measurement procedure was initiated by first attaching the device to the participant and activating the power supply. After boot-up, $10 \mathrm{~s}$ elapsed before data acquisition commenced in a stable transfer mode. Subsequently, the participants were moved to an asphalt walking street (Fig. 2) to obtain the measurements described above. The participant stopped walking, and the power supply was cut after the time specified by the control unit had elapsed. The above procedure was performed for a single measurement, and 15 measurements were collected per person. During the measurement, each participant was directed to step on to the walking path, starting with the right foot, and to adjust their stride and cadence to $100 \mathrm{steps} / \mathrm{min}$. [13-17].

One of two conditions existed on the walking path-a dry road surface and a frozen road surface. The dry road surface was defined as the section where no ice layer was present, visually appearing as a wet road surface, in which an adequate walking distance was secured. The frozen road surface was defined as the range covered by snow and ice layers with a density of $0.75 \mathrm{~g} / \mathrm{cm}^{3}$ or greater [18]. The total length of the walking path was $10.00 \mathrm{~m}$, and an additional support distance $(3.25 \mathrm{~m})$ and follow-up distance $(3.50 \mathrm{~m})$ were secured [19-20]. The entrance of a medium-sized facility was used for the measurement. The measurements were carried out after sunset (18:00), when accidental falls frequently occur. The outside temperature was approximately $-4{ }^{\circ} \mathrm{C}$, with humidity near $80 \%$. A thermal-transmission type measuring device (EDX-10B, EDX-13A, sampling frequency: $1 \mathrm{kHz}, \mathrm{KYOWA})$ was used to provide temperature control of the freezing conditions. 


\section{E. Analytical Method}

The acquired data were processed in Excel (Microsoft Company, ver. 2016) and analyzed using the statistical analysis software R (ver.3.5.0). A comparison was made between the data obtained on the dry surface of the walking path and the frozen surface. The data parameters were associated with the swing phase of the unit-mounting side. The purpose of this study is to provide a more effective fall prevention technique by detecting the road condition before the participant steps on the road surface. Because the position of the target road surface on the walking road and the stride of the participant were fixed, the expectation exists that the measurement of the target road surface would be performed simultaneously with the statistical processing. Therefore, because the detection of the target road surface was performed between the middle and second half of the swing phase, statistical processing was also performed at this time. The data were used to perform multiple comparison tests using the Steel-Dwass method to consider normality.

\section{RESULTS}

\section{A. Measurement Result of Reflected Light}

The resulting amount of light reflected from the road surface between the swing and the stance phase is shown in Fig. 3. The vertical axis of the graph depicts the acquired value (in arbitrary units: a.u.), representing the amount of light. The horizontal axis denotes the gait cycle ( $0 \%$ to $40 \%$ indicates the swing phase, $60 \%$ to $100 \%$ indicates the stance phase). The graph in Fig. 3 demonstrates the differing trends in the output values between the dry and frozen road surfaces from approximately $20 \%$ of the gait cycle (depicted with a dotted arrow in the figure). From the $40 \%$ walk phase onwards, the light value obtained for the dry road surface increased rapidly, while the corresponding value for the frozen road surface was confirmed to be moderate. This trend continued until $60 \%$ of the gait cycle (mid-stance). After this, until the $100 \%$ walk phase, a decreasing tendency in this value was observed.

The statistical processing described above indicated a significant difference in the results between the dry and frozen surfaces during the $25 \%$ to $40 \%$ walk phases, as reported in Table 2. Hence, each road surface exhibited different tendencies during the swing phase, which can be observed in the measurement scan.

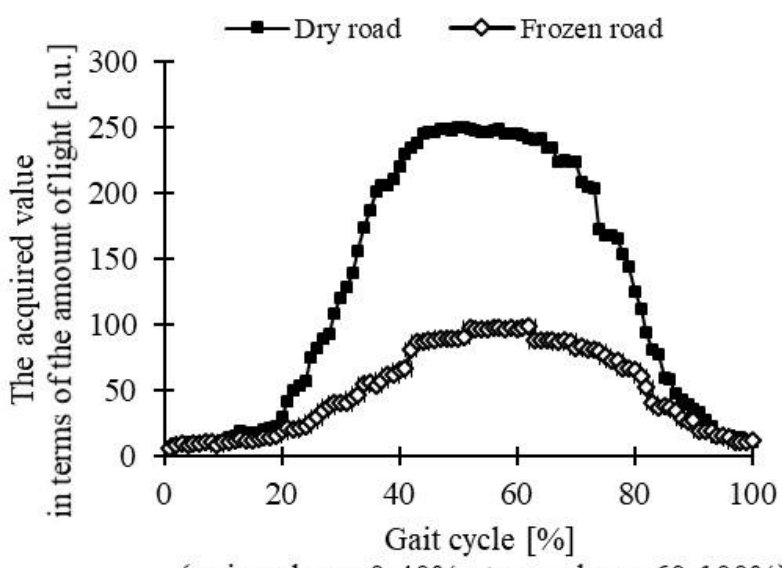

(swing phase: $0-40 \%$, stance phase: $60-100 \%$ )

Fig. 3 Measurement result of reflected light

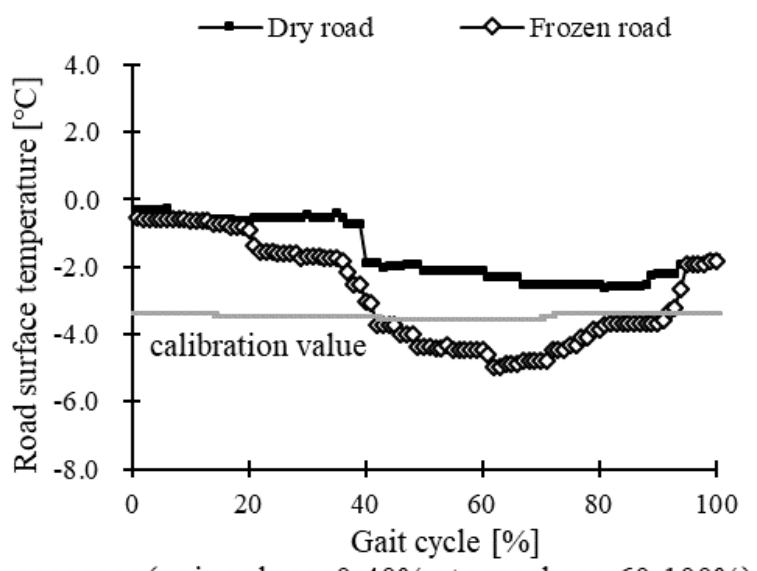

(swing phase: $0-40 \%$, stance phase: $60-100 \%$ )

Fig. 4 Measurement result of road surface temperature

\section{B. Measurement Result of Road Surface Temperature}

The results of the road surface temperature are displayed in Fig. 4. The vertical axis of the graph shows the road surface temperature $\left({ }^{\circ} \mathrm{C}\right)$, and the horizontal axis depicts the gait cycle (\%). The graph in Fig. 4 shows that different trends appeared in the temperature between the dry and frozen road surfaces beginning at approximately $20 \%$ walk phase (depicted by a dotted arrow in the figure). Based on the statistical analysis described above, significant differences were observed between the dry and frozen surfaces during the $30 \%$ to $40 \%$ walk phase, as listed in Table 2 . The road surface

Table 2 Result of amount of reflected light and road surface temperature (only mid swing - terminal swing phase)

\begin{tabular}{|c|c|c|c|c|c|c|c|c|c|c|}
\hline \multicolumn{6}{|c|}{ Amount of reflected light [a.u.] } & \multicolumn{5}{|c|}{ Road surface temperature $\left[{ }^{\circ} \mathrm{C}\right]$} \\
\hline \multirow{2}{*}{$\begin{array}{c}\text { Swing } \\
\text { phase[\%] }\end{array}$} & \multicolumn{2}{|c|}{ Drying road } & \multicolumn{2}{|c|}{ Freezing road } & \multirow{2}{*}{$\begin{array}{c}\text { Steel-Dwass } \\
\text { test }\end{array}$} & \multicolumn{2}{|c|}{ Drying road } & \multicolumn{2}{|c|}{ Freezing road } & \multirow{2}{*}{$\begin{array}{c}\text { Steel-Dwass } \\
\text { test }\end{array}$} \\
\hline & Avg. & S.D. & Avg. & S.D. & & Avg. & S.D. & Avg. & S.D. & \\
\hline 20.0 & 41.03 & 29.90 & 21.20 & 8.39 & - & -0.62 & 1.28 & -0.88 & 3.76 & - \\
\hline 25.0 & 81.76 & 62.43 & 29.16 & 19.64 & $* *$ & -0.53 & 1.44 & -1.59 & 3.66 & - \\
\hline 30.0 & 128.97 & 82.92 & 39.80 & 28.95 & $* *$ & -0.43 & 1.45 & -1.70 & 3.61 & $* *$ \\
\hline 35.0 & 201.45 & 111.12 & 53.96 & 38.79 & $* *$ & -0.38 & 1.30 & -1.73 & 3.64 & $* *$ \\
\hline 40.0 & 230.07 & 116.49 & 66.12 & 37.28 & $* *$ & -1.87 & 2.23 & -3.04 & 4.50 & ** \\
\hline
\end{tabular}




\section{Development of A Road Surface Discrimination Device for Preventing Falls during Winter Walking - Built-In to Shoes and Verification on Frozen Surfaces -}

temperature results also tended to reach different measurement values during the swing phase on each road surface.

Moreover, the temperature value obtained for the frozen road surface by the unit was compared with the calibration value of the measuring device used for temperature control. The unit value and the calibration value matched after heel contact (40\% walk phase or later), and consequently, the measurement of the actual frozen surface temperature was timed after the heel contacted the road surface. Unlike the calibration value, the value obtained by the unit was variable, tending to rise after a period of descent.

\section{DISCUSSION}

\section{A. Attenuation of Reflected Light}

The output values representing the amount of reflected light for the dry and frozen surfaces exhibit significant differences. These differences are due to the absorbance characteristics of the infrared sensor in the manufactured unit and the presumed influence of the distance (height) between the shoe and the road surface. The light emitted from the measuring device diffuses; however, when the distance (height) between the shoe and the road surface is small, the light reflected on the road surface tends to enter the detection range of the unit (Fig. 5).

In the results obtained for the dry road surface (Fig. 3), the amount of reflected light increased as the shoe gradually approached the road surface from the swing phase until heel contact $(40 \%)$. Furthermore, from the mid-stance to the terminal stance $(60 \%-100 \%)$, the amount of light decreased as the heel moved away. Therefore, the tendency observed in the results presumably appeared owing to the differences in the distance (height) between the shoe and the road surface.

In contrast, in the results for the frozen road surface (Fig. 3 ), this type of tendency was minor. This is presumably due to the influence of the absorbance characteristic of the wavelength used in the unit. The light irradiated by the light source of the unit is easily absorbed by water and ice. Therefore, when there was ice or water film on the road surface, the measured amount of light most likely did not reach the value obtained for the dry road surface owing to attenuation (Fig. 5). This tendency was also noticeable even after heel contact ( $40 \%$ to $100 \%$ ). Therefore, the amount of
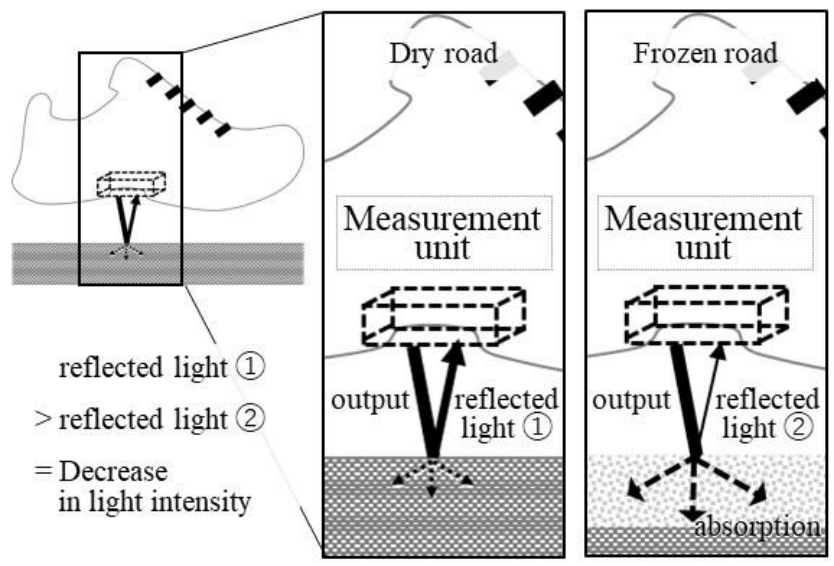

Fig. 5 Attenuation mechanism of reflected light light acquired increased from the middle of the swing phase to the end of the stance phase during walking on the dry road surface. In contrast, the amount of light in these phases increased only slightly during the walking cycles on the frozen road surface. The trends observed between each trial and between participants confirmed that variation exists in the amount of light obtained based on the differences in the direction of the shoe approach, from the toe-off stance during walking to the initial swing stance.

\section{B. Temperature Measurement on Frozen Road (while walking)}

The results of the road surface temperature (Fig. 4) demonstrate that a significant difference exists in the value acquired for the dry and frozen road surfaces $(\mathrm{p}<0.01)$. A conceivable explanation is that the measurement range of the temperature sensor in the measuring device corresponds to the set road surface temperature. Naturally, the set temperature range under experimental conditions is fully covered, even when compared to the standard of the sensor used for the measurement device. Therefore, the employed sensor was presumably able to acquire the road surface temperature, even under irregular conditions such as walking. Moreover, the tendency shown in Fig. 4 was confirmed, despite the visualization of the results of each participant.

\section{Variations of Acquisition Temperature}

The temperature value obtained by the unit for the frozen road surface matches the calibration value after heel contact ( $40 \%$ or later); however, this value is not constant. This result could be due to the irregular measurement conditions influencing the data collection during the walking action. Under normal conditions, a radiation temperature sensor measures the temperature while the sensor or object remains at a fixed location, and the measurement is performed at a constant distance and interval. However, in this study, the measurements were carried out during the course of walking; thus, the measurement distance and orientation of the sensor

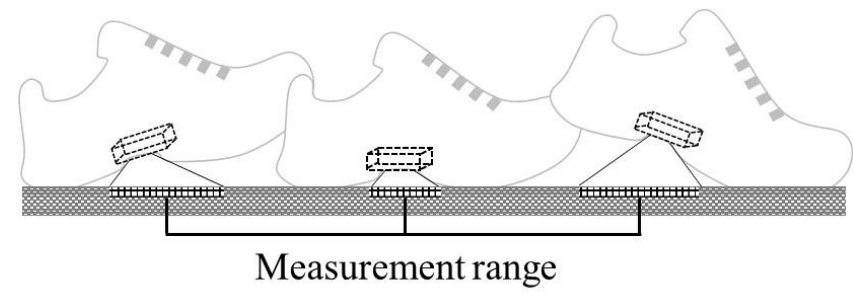

Fig. 6 Measurement range during walking
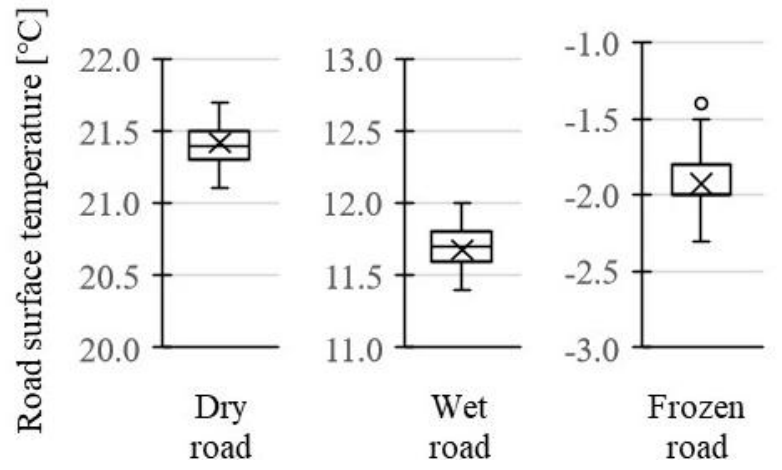

Fig. 7 Measurement result of road surface temperature (sensor fixed) 
were constantly changing (Fig. 6). Therefore, it is presumed that the observed variations in the acquisition temperature appeared because of the increased likelihood of a larger error than that given by the measurement accuracy, guaranteed by the standard of the sensor.

In addition, the measured temperature using the temperature sensor was confirmed at a fixed point in a preliminary experiment; however, the variation in the acquisition temperature was confirmed to be small compared with the measurements conducted in this study (Fig. 7). Therefore, in the walking measurements, it is presumed that the acquired temperature varied greatly, relative to the calibration value, even when considering the tolerance about the standard.

\section{CONCLUSIONS}

In conclusion, this study devised a road surface perception system for pedestrians with the aim of preventing falls during the winter period. A previously manufactured unit capable of obtaining road surface information on sidewalks was evaluated on an actual frozen road surface in the current study. The results indicate that measuring the amount of light and road surface temperature under average walking conditions is possible. Moreover, results suggest that the measurements acquired by the unit might be effective for assessing the road surface during walking, as differences were observed between the acquired values for each road surface. In the future, we would like to increase the number of units and samples, and further demonstrate the measurement accuracy and its usefulness. Moreover, we wish to examine the results obtained under different walking and road surface conditions.

\section{ACKNOWLEDGMENT}

The authors thank the subjects for their participation.

\section{REFERENCES}

[1] Teruyuki F, Hiroshi W, Akihiro F, "Generating Mechanism of Road Icing Phenomenon,” Journal of Snow Engineering, vol.21, 2005 pp.110-115.

[2] Yoshinobu Y, Fumie M, Katsumi H, et al., "An Analysis of Fall Accidents in Japan Based on Fire Department Ambulance Statistics: Investigation of Time and Location," Japanese Journal of Public Health, vol.57, 2010, pp.403-409.

[3] Toshimichi S, Takahiro G, Seiichi M, et al., "Examination Using TUGT Concerning Physical Strength Level of Elderly People Who Can Go Out Even in Snowfall," Rigakuryoho Kagaku, vol.20, 2005, pp.179-182.

[4] Hiroaki F, Noriko T, Kazumi A, et al., "Physical Therapy for Transcervical Fracture in the Elderly," Rigakuryoho Kagaku, vol.17, 2002, pp.149-156.

[5] Masakazu I, Takeyuki Y, Kazuhiro S, et al., "Comparison of Young and Elderly Adults in a Standing-walking Task," Rigakuryoho Kagaku, vol.32, 2017, pp. 221-225.

[6] Kazufumi A, Yuji N, Yasuhiro T, et al., "Standing Body Balance and Fall Related Factors in Community-Dwelling Elderly Persons," Rigakuryoho Kagaku, vol.23, 2008, pp.597-600.

[7] Nagata Y, Kaneda Y, Tomita M, et al., "Sapporo Citizens' Efforts to Prevent Slip and Fall Accidents in Winter," Annual Report on Snow and Ice Studies in Hokkaido, vol.34, 2015, pp.75-78.

[8] Yoko S, Fumihiro H, Tetsuo A, et al., "Pedestrian Slip and Fall Accidents in the Sapporo City Center in Winter," Comprehensive Urban Studies, vol.85, 2005, pp.57-68.
[9] Keisuke I, Atsushi N, Motoko I, et al., "Effect on Partial Weight Bearing of Acoustic Alarm Feedback Provided by Portable Electronic Foot Sensor Shoes (Step Aid®),” Rigakuryoho Kagaku, vol.30, 2015, pp.445-448.

[10] Satoshi I, Keisuke K, Hiroki M, et al., "Development of a Shoe Built-in Road Surface Discrimination Device for Prevention Fall in Winter Examination on the Simulated Winter Road -," Bulletin of the Japanese Society of Prosthetic and Orthotic Education, Research and Development, vol.36, 2020(in press).

[11] Mutsuo I, Sumio K, Jun U, Kinsekigai Bunkoho Nyumon. Tokyo, 1994, pp.45-47.

[12] Daisuke I, Hiroshi K, Akihiro K, et al., "Measurement of Surface Roughness of Icy Road Surface by Spectral Polarimetry," Transactions of the Society of Automotive Engineers of Japan, Inc., vol.46, 2015, pp.931-936.

[13] Donald A N, Kinesiology of the Musculoskeletal System: Foundations for Rehabilitation. Tokyo, 2016, pp.696-697.

[14] Kotoko S, Hitoshi M, "Relation of Agility to Walking Ability Comparison of the Young and the Middle-Aged -," Rigakuryoho Kagaku, vol.21, 2006, pp.7-11.

[15] Hironori O, Kayoko M, Kenta S, "Characteristics of Backward Walking of Healthy Adult," Rigakuryoho Kagaku, vol.22, 2007, pp.199-203.

[16] Yuko I, Akira K, "Relationships between Body Composition and Gait Speed in Young Females," Rigakuryoho Kagaku, vol.34, 2019, pp.473-477.

[17] Tadayoshi A, Hiroshi S, Naoki Y, et al., "Movement Control in the First Step of Gait Initiation on a Low Friction Floor," Rigakuryoho Kagaku, vol.17, 2002, pp.49-52.

[18] Seiiti K, Eizi A, "Snow and Ice on Roads I. Low Temperature Science," Physical Sciences, vol.27, 1970, pp.163-179.

[19] Asami K, Hideyuki N, Hitoshi T, "Preliminary Study of Distance needed to Achieve Constant Walking Speed and Step Length by Young Healthy Persons," Rigakuryoho Kagaku, vol.26, 2011, pp.647-650.

[20] Hyuma M, Tsutomu A, Shinichi F, et al., "Examination of Gait Measurement in Home-Based Rehabilitation: Gait Ability Evaluation Using a 1.5 Meters Walking Test," Rigakuryoho Kagaku, vol.20, 2005, pp.203-206.

Satoshi Izumiya is a graduate school student (Ph.D.). He is a prosthetist and orthotist and has a M.S. (Engineering). He is interested in medical and engineering, working on the Fall prevention devices development.

Prof. Keisuke Kon, Ph.D.(Health Science). He is a highly skilled prosthetist and orthotist. He is currently working, Hokkaido University of Science.

Prof. Hiroki Matsuzaki, Ph.D.(Engineering). He is a professional of sounds. He is currently working, Hokkaido University of Science.

Prof. Tamotsu Mitamura, Ph.D.(Engineering). He is a professional of Information Technology. He is currently working, Hokkaido University of Science. 